\title{
Modified TAROT for cross-selling personal financial products
}

\begin{abstract}
The Top Application characteristics Remainder Offer characteristics Tree (TAROT) was first introduced in 2007. This is a modified Classification and Regression Trees (CART) used to help decide which question(s) to ask potential applicants to customise an offer of a personal financial product so that it would have a high probability of take up. In this piece of work the authors are presenting, they have further modified the TAROT to cross TAROT, using its properties and modeling steps to deal with the issue of cross-selling. Since the bank already has ready customers, it would be ideal to cross-sell the financial products seeing that one can ask one (or more) further question(s) based on the initial offer to identify and customise another financial product to offer.
\end{abstract}

Keyword: CART; Acceptance probability; Cross-selling 